\title{
FIXED PROSTHETIC RESTORATION, SUPPOR- TED BY NATURAL TEETH AND SIP TITANIUM IMPLANT. (A 14-years survey)
}

\author{
Metodi Abadzhiev \\ Prosthodontic Department, Faculty of Dental Medicine, \\ Medical University - Varna
}

\section{SUMMARY:}

The success of the implantologic treatment depends on many factors of surgical, prosthetic and prophylactic nature. The kind of the implantologic system is of particular importance so far as it fits to the specific clinical case (the kind of the prosthetic restoration, the bone type). The combined support, both on natural teeth and implants, is criticized by a number of authors. The most impartial assessment of the functional suitability of the implantologic treatment however, is the time proof.

Key words: implants, SIP

\section{PURPOSE:}

This study shows a long (14 years) functional suitability of a bridge on a combined support, both natural teeth and an implant.

\section{INTRODUCTION:}

There are publications about using combined support, natural teeth and implants, in the literature. But the current rules for planning and building of the prosthetic restoration on implants do not recommend combined support. There are clinical cases at which this approach is possibly the minimum invasive one, and the idea of minimum invasivity is one of the contemporary tendencies in implantology.

\section{METHODS AND MATERIALS:}

A 49-years old patient, female. In 1995 one implant is placed in 37region. The followed surgical protocol is that of the SIP-system. The metal-ceramic bridge restoration is on combined support -natural teeth 34, 35 and implant 37. The teeth have no periodontal problems, pathologic mobility or inflammation signs. They are endodontically treated. The patient hasn't visited the dental practice for prophylaxis for more than 8 years.

\section{RESULTS:}

The patient visits the dental practice for the first time 8 years after the placement of the implant and its loading the reasons are fractured ceramic coating of the bridge and caries of all teeth. All the remaining teeth are put to endodontic and prosthetic (metal-ceramic crowns) treatment. (fig. 1.) The oral hygiene level of the patient is high. 6 years later however, begin problems with the non vital teeth, due to their imprecise treatment. There is no X-ray or clinical evidence about bone resorption around the implant.

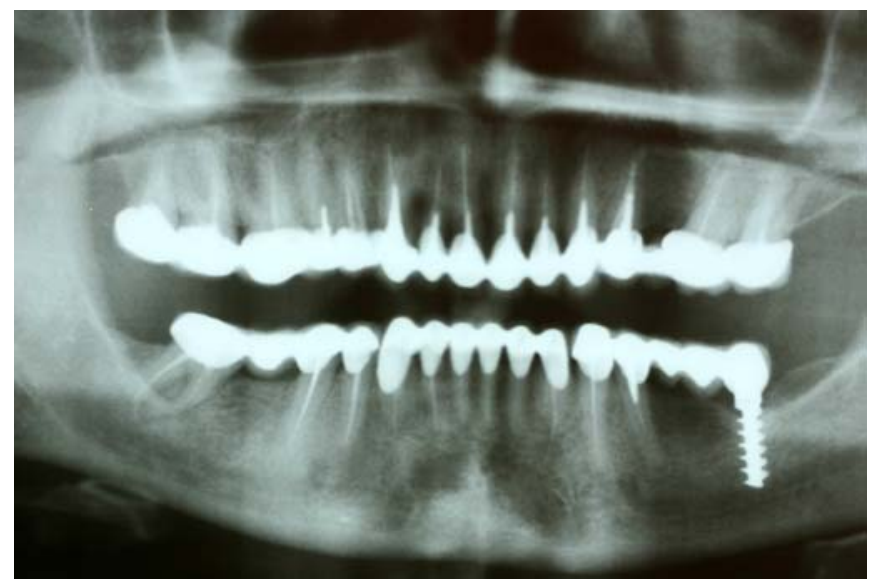

Fig. 1. OPG after completion of the endodontic and prosthetic treatment- 8 years after the placement of the implant

\section{DISCUSSION:}

The presented clinical case is not a rule's exception. Combining natural teeth and implants as a support is a smart decision at patients, which dentition is periodontally healthy, but predisposed to caries. This saves us from placing many implants. In cases like this, should be followed the rule for prevailing of the supports of one kind and placing of bridges with reduced forms. The significant for this clinical case is that the endodontically treated teeth are functionally suitable less time than the implants, used as a support for extension of the tooth row together with natural teeth. 


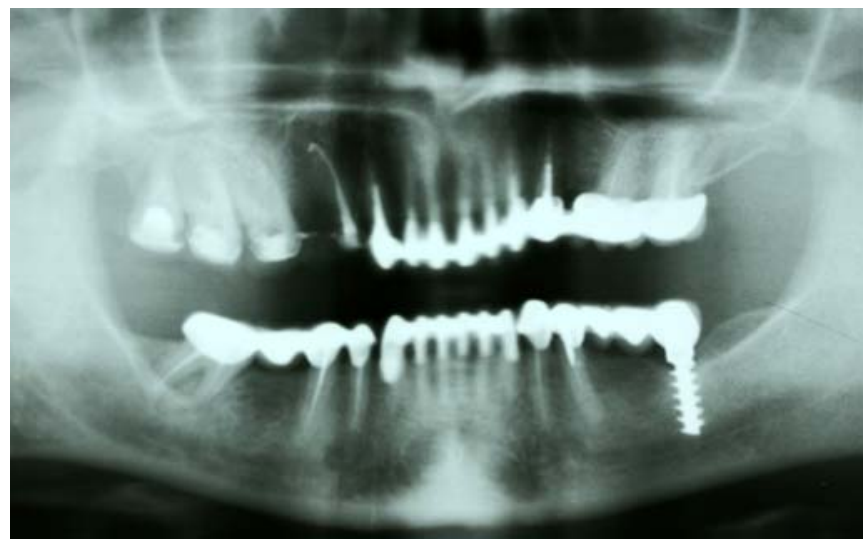

\section{CONCLUSIONS:}

The individual approach to every case is very important in implantology. Bulgarian implantologic school is richly experienced in combining natural teeth and implants as a support for fixed restorations. The main principles of the combined support can be resumed as follows:

- Combining of supports only in periodontally healthy patients.

- Including teeth, belonging to adjacent groups.

- Prevailing of implantologic or periodontal block.

- Reducing of the vestibular-lingual dimension of the bridge parts.

Fig.2. The OPG after extraction of 15; - 14 years after the placement of the implant

\section{REFERENCES:}

1. Intrusion of teeth in the combination implant-to-natural-tooth fixed partial denture: a review of the theories. Pesun I. J. J Prosthodont. 1997 Dec;6(4):268-77

2. Splinting osseointegrated implants and natural teeth in rehabilitation of partially edentulous patients. Part I: laboratory and clinical studies. Gross M., Laufer B. Z. J Oral Rehabil. 1997 Nov; 24(11):863-70

3. Intrusion phenomenon in combination tooth-implant restorations: a review of the literature. Schlumberger T. L., Bowley J. F., Maze G. I. J Prosthet Dent.
1998 Aug;80(2):199-203

4. Histologic evaluation of the periodontium of abutment teeth in combination implant/tooth fixed partial denture. Pesun I. J., Steflik D. E., Parr G. R., Hanes P. J. Int J Oral Maxillofac Implants. 1999 May-Jun;14(3):342-50.

5. Photoelastic stress analysis of load transfer to implants and natural teeth comparing rigid and semirigid connectors. Nishimura R. D., Ochiai K. T., Caputo A. A., Jeong C. M. J Prosthet Dent. 1999 Jun;81(6):696-703.

6. Implant Dentistry, Popov N, Index,
1999.

7. Yordanov B. - Prosthetic Treatment With Implant Bridge Restorations (Investigations in Implant - Natural ToothDental Bridge System), Disertation, Faculty of Dental Medicine, 2004.

8. Biomechanical interactions in toothimplant-supported fixed partial dentures with variations in the number of splinted teeth and connector type: a finite element analysis. Lin C. L., Wang J. C., Chang WJ. Clin Oral Implants Res. 2008 Jan; 19(1):107-17. Epub 2007 Oct16.

Address for correspondence:

Metodi Abadzhiev

Prosthodontic Department, Faculty of Dental Medicine, Medical University - Varna

63, G. S. Rakovski Str., 9000 Varna, Bulgaria

E-mail:mabadjieff@yahoo.com 